\title{
Verification of the stability lobes of Inconel 718 milling by recurrence plot applications and composite multiscale entropy analysis
}

\author{
Kecik Krzysztof ${ }^{\mathrm{a}}$, Marek Borowiec ${ }^{\mathrm{b}}$, and Rusinek Rafał ${ }^{\mathrm{c}}$ \\ Lublin University of Technology, Nadbystrzycka 36, 20-618 Lublin
}

Received: 28 November 2015

Published online: 15 January 2016

(c) The Author(s) 2016. This article is published with open access at Springerlink.com

\begin{abstract}
Correctness verification of the stability lobe diagrams of milling process determined by commercial software CutPro 9 is the aim of this work. The analysis is performed for nickel superalloy Inconel 718 which is widely used in aviation industry. A methodology of stability analysis which bases on advanced nonlinear methods such as recurrence plot, recurrence quantifications analysis and composite multiscale entropy analysis are applied to the experimental data. Additionally, a new criterion for the determination of the unstable areas is proposed.
\end{abstract}

\section{Introduction}

Recently nickel and titanium alloys have become very good for the construction of aircrafts parts, and medical and chemical processing industries. Generally, it is known that nickel-based superalloys are one of the most difficult materials to machine, mainly due to fast tool wear. High-speed machining is economically well founded where machine centers can operate at spindle speeds as high as half a million revolutions per minute $[1,2]$. Therefore, vibrations occurring during machining can generate a serious problem for engineers. Undesired relative vibrations between the tool and the workpiece may deteriorate the quality of machined surfaces or even damage the machine tool and scrap the workpiece. Then cutting forces which depend on the tool geometry, material properties, feed rate and cutting speed can have large amplitude. When a process is unstable, the amplitude of vibrations may grow exponentially. These unstable vibrations of the tool, known as chatter, can create large cutting forces. This is an undesired phenomenon, because the surface of the workpiece becomes nonsmooth as a result of significant vibrations of the cutter. Moreover, the cutting tool wears out rapidly when chatter exists.

In order to use a full capacity of a new machine and also to achieve potentially high material removal rate together with the desired surface quality, optimum machining parameters are necessary. One of the parameters which improve the efficiency of the cutting process is the cutting depth and rotational speed. Usually, the choice of cutting depth and rotational speed is based on a Stability Lobes Diagram (SLD). Stability diagrams can be applied in a high-speed machining processes to optimize the maximum depth of cut at the highest available spindle speed. Using high-speed machining, an increase of material removal rates is achieved through a combination of large axial depths of cut and high spindle speeds. When the cutting depth exceeds the critical value chatter vibrations can arise at some spindle speed, whereas, if the cut depth is below the critical value, the cutting is stable regardless of the spindle speed. In many practical cases, the choices of the optimal speed and of the depth of cut are difficult because of the limit set by vibrations that arise during the material removal process [3] and experience is needed in modal analysis of a tool-spindle system.

Many papers show the modeling of the milling process on the basis of which the stability diagrams are determined (for example [4-7]). Then SLD are obtained by simulation in the time domain equation of motion. The alternative solution is to calculate the stability lobes directly in the frequency domain or to resolve the delay-differential equations (DDE [8]). However, only a few papers present complete experimental verification of these stability lobes [9].

\footnotetext{
${ }^{a}$ e-mail: k.kecik@pollub.pl

b e-mail: m.borowiec@pollub.pl (Corresponding author)

${ }^{\text {c }}$ e-mail: r.rusinek@pollub.pl
} 


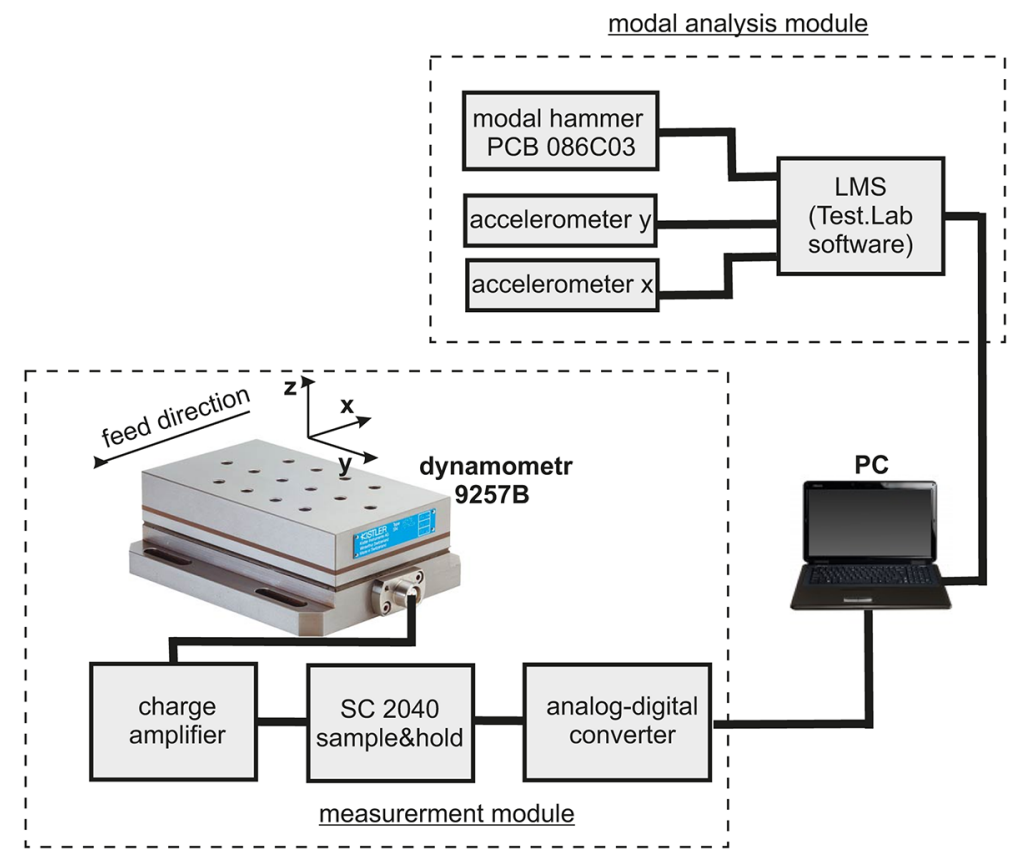

Fig. 1. Cutting force and modal analysis modules of the experimental system.

This work shows the experimental results of milling of Inconel 718 with continuously growing depth of cut for a fixed spindle speed. This way of the machining should demonstrate pass from stable milling to unstable. Next, the verification of process stability based on two new nonlinear methods: recurrence plot, recurrence quantifications [10,11] and entropy is shown [12]. Stability of the milling process is practically hardly distinguished looking only at the force time histories and its magnitude. Therefore, this analysis is extended to more advanced tools. Additionally, the recurrence plot quantifications to process stability classification is proposed.

\section{Description of the experimental setup}

The measurements are conducted on the laboratory equipment, presented schematically in fig. 1, which is composed of the numerical controlled milling machine FV580A, the piezoelectric dynamometer Kistler 9257B, the charge amplifier type 5017B, the "sample and hold" component (SC2040) and the analog-digital converter NI 6071E. Additionally, the LMS module controlled by TestLab software with the two piezoelectric accelerometers PCB $352 \mathrm{~B} 10$ in order to measure vibrations acceleration in $x$ and $y$ directions and modal hammer PCB model 086C03 is used to perform modal analysis.

At this stage, an instrumented modal hammer is engaged to excite the tool at its free end (i.e., the tool point) and the resulting vibrations are measured using a low mass accelerometer mounted at the tool point.

In the second stage of the experiment the cutting forces $\left(F_{x}, F_{y}\right.$ and $\left.F_{z}\right)$ generated on the workpiece during the machining were measured by the dynamometer mounted on the milling machine. Next, the force signals are transmitted to charge amplifier next to the module sample\&hold and finally to the analog-digital converter, which is connected to the computer. The sampling rate of data reordered during the test equals $5 \mathrm{kHz}$. The material used in the experiment is Inconel 718. In the tests the solid carbide end mill F4BT1200AWX45R100 (Kennametal) with a diameter of 12 mm and 4 cutting edges is used during down milling. The radial depth of cut is two thirds of the end mill diameter ( 8 mm).

\section{Modal and stability analysis}

\subsection{Frequency response function}

A modal analysis is the process of determining the dynamic characteristics of a system in forms of natural frequencies, damping factors and mode shapes as frequency response function results. Then the modal parameters can used to formulate a mathematical model. This technique describes the relationship between the system response at one location and excitation at the same or another location as a function of the excitation frequency. This relationship, which is often a complex mathematical function, is known as frequency response function (FRF). The FRF consists of two parts:

- the real part of the FRF versus frequency (the black line in fig. 2),

- the imaginary part of the FRF versus frequency (the grey line in fig. 2). 

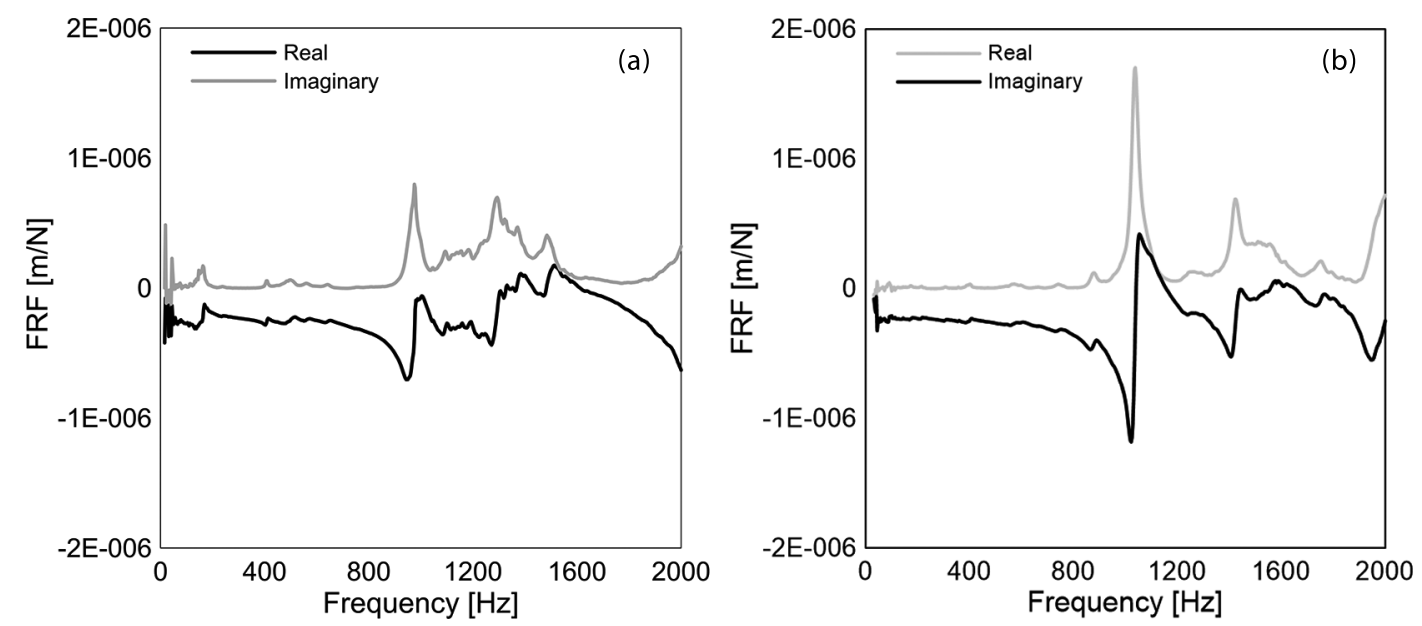

Fig. 2. Frequency response function (FRF), for $x$ (a) and $y(\mathrm{~b})$ directions.
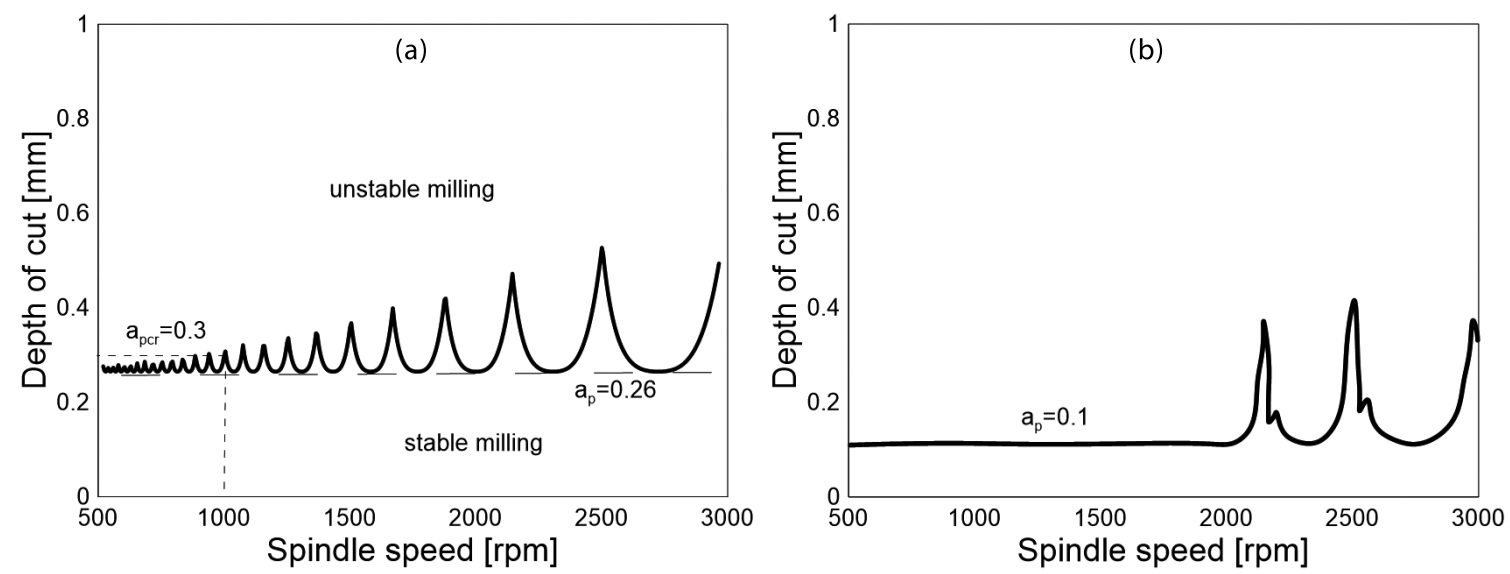

Fig. 3. Stability lobe diagram determined by the software CutPro9 (a) and the package "machinist online" [13] (b).

The FRF is obtained by impact tests, where the instrumented modal hammer is used to excite the tool and the resulting vibrations are measured by the low-mass accelerometers mounted at the tool point. Using the LMS TestLab software, the structural dynamic parameters of a tool spindle system from FRF are calculated.

The frequency response function for the end mill mounted in the holder is determined in the $x$-direction (fig. 2a) and $y$-direction (fig. 2b). The natural frequency of the spindle system given by FRF is about $1 \mathrm{kHz}$.

\subsection{Stability lobe diagram}

The damping and stiffness can be calculated from FRF with the help of the commercial software CutPro9 in order to generate the corresponding stability lobe diagram (fig. 3a). For comparison the SLD was also obtained by the package "machinist online" via the Internet [13], (fig. 3b). This application enables the user to produce stability lobe diagrams of the tool and holder system. Comparing the two diagrams we observe significant differences in the critical depth of cut $\left(a_{p}\right)$ at which chatter exists. In experimental modal analysis the critical axial depth of cut equals $0.26 \mathrm{~mm}$, in the package "machinist online" $0.1 \mathrm{~mm}$. This is because, the modal analysis is more precise than the package "machinist online" which is based only on the geometry of a tool holder, milling parameters and the material data.

Stable cuts should occur in the region below the stability boundary (below the black line, fig. 3a), while unstable cuts should occur above the stability boundary. As shown, it is possible to increase the axial depth of cut without chatter by choosing the proper spindle speed which omits unstable lobes. This simple method of parameter selection can improve productivity. Our aim is to check the correctness of the stability lobe diagram by analyzing the cutting forces with the help of advanced nonlinear methods as the recurrence plot and the recurrence quantification analysis. 

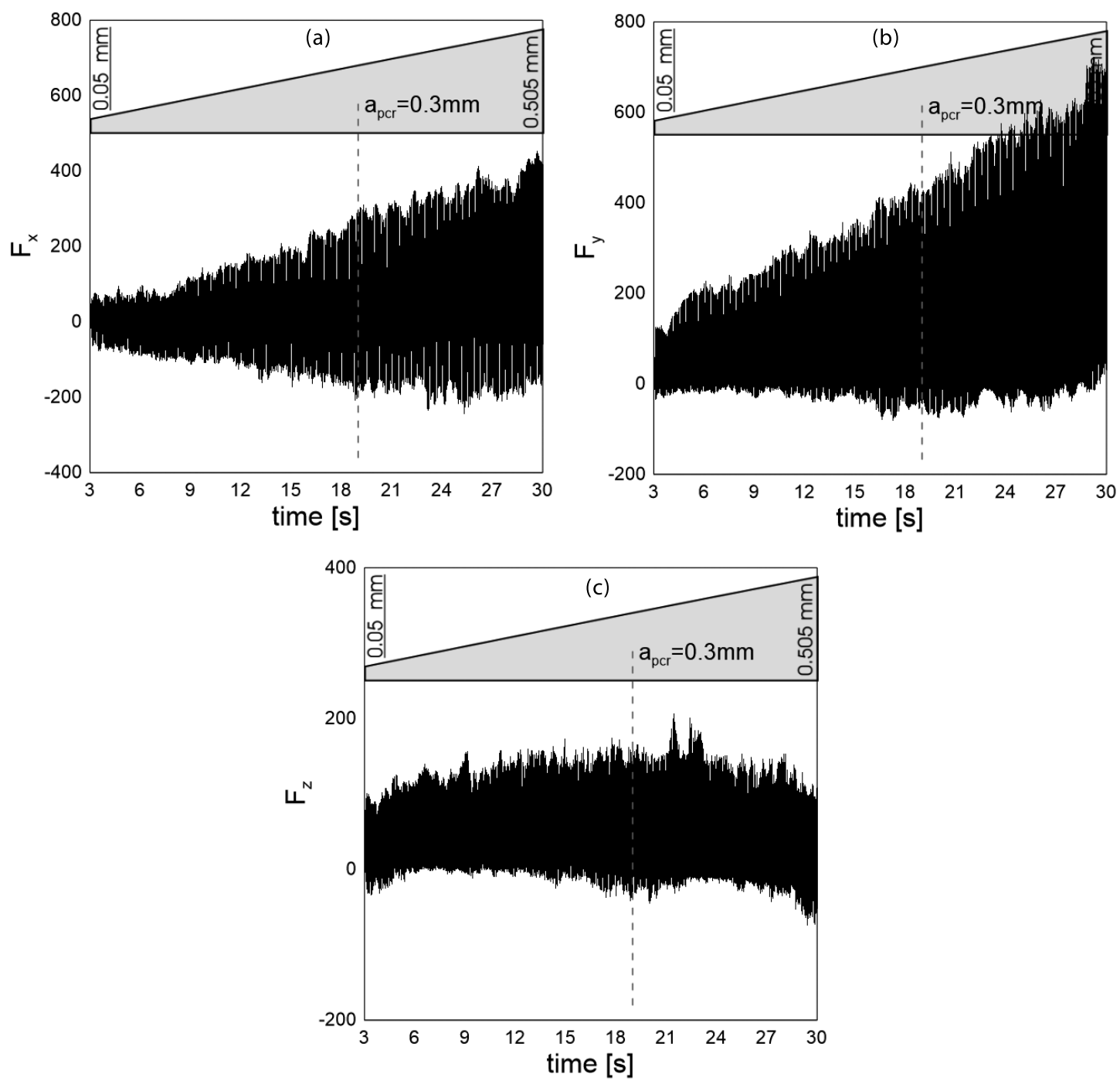

Fig. 4. Cutting forces $F_{x}(\mathrm{a}), F_{y}(\mathrm{~b})$ and $F_{z}$ (c) of Inconel 718 milling with the fixed spindle speed $n=1000 \mathrm{rpm}$.

\section{Experimental results}

\subsection{Milling with increasing depth of cut}

Analyzing SLD result (fig. 3a), the cutting depth below $0.26 \mathrm{~mm}$ should give stable cutting all the times regardless of the spindle speed. Therefore the experiment is performed, for continuously increasing depth of cut (from 0.05 to $0.505 \mathrm{~mm}$ ) for fixed spindle speed $n=1000 \mathrm{rpm}$ and is presented in fig. 4 . The time series of the three components of the cutting forces $F_{x}, F_{y}$ and $F_{z}$ are presented.

The forces $F_{x}$ (force in the feed direction, fig. 4a) and $F_{y}$ (fig. 4b) have similar trends, but the values of the force $F_{x}$ are smaller in comparison to $F_{y}$.

Force $F_{z}$ (axial end mill direction) is not analyzed further due to the fact that its value is almost constant in all the analyzed ranges of depth of cut. That is caused by strong tool stiffness in the $z$-direction compared to that in $x$, and $y$ directions. Therefore, for further research only the force $F_{x}$ or $F_{y}$ is considered. To investigate the cutting force signal and to find symptoms of the stability loss, the recurrence plot technique (RP) is applied for representative $F_{x}$ force.

\subsection{Recurrence plot analysis (RP)}

The RP approach provides a qualitative interpretation of the hidden patterns of the dynamical systems, based on phase space reconstruction which begins with a time delay embedding of the data. This technique was originally introduced by Eckmann [11]. 

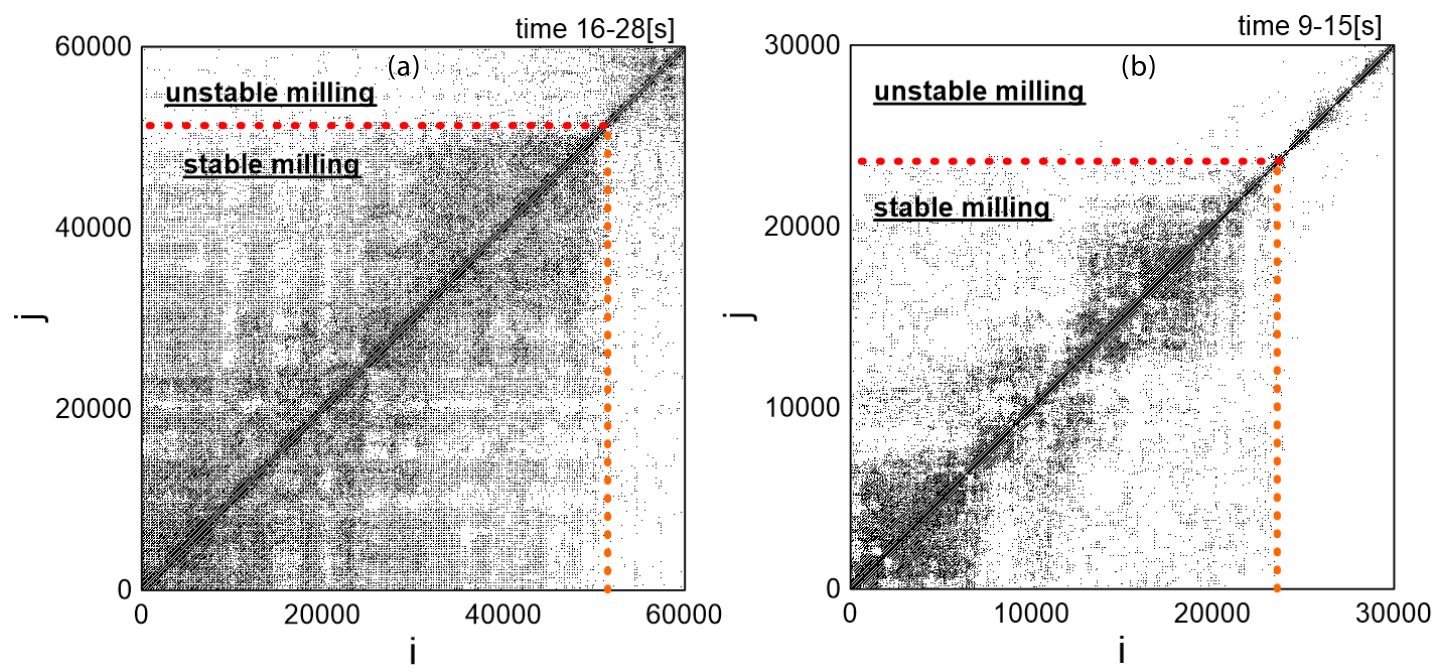

Fig. 5. Recurrence plot for $n=1000 \mathrm{rpm}, d=27, m=5, \varepsilon=10$ (a) and $n=2000 \mathrm{rpm}, d=15, m=5$ and $\varepsilon=25$ (b).

A recurrence plot is a visualization of a diagram (square matrix), in which the points correspond to those times at which a state of a dynamical system recurs. Technically, the RP reveals all the times (both axes $i$ and $j$ are time axes) when the phase space trajectory of the dynamical system visits roughly the same area in the phase space. A recurrence plot can be described by computing the matrix

$$
R P_{i j}=\theta\left(\varepsilon-\left|\mathbf{x}_{i}-\mathbf{x}_{j}\right|\right)
$$

$\boldsymbol{x}_{i}$ is the delay vector, calculated from

$$
\mathbf{x}_{i}=\left(x_{i}, x_{i+d}, x_{i+2 d}, \ldots, x_{i+(m-1) d}\right) .
$$

The parameters $m, d$ and $\varepsilon$ denote an embedding dimension, a time delay and a threshold, respectively. The detailed description of recurrence plot and methods for selecting embedding parameters $m, d$ and $\varepsilon$ can be found in the papers $[14,15]$.

In fig. 5a the recurrence plot of the force $F_{x}$ for the period of time 16-28s, where the system passes through a stability boundary, at spindle speed $n=1000 \mathrm{rpm}$ is presented. While fig. $5 \mathrm{~b}$ presents RP diagram obtained for spindle speed $n=2000 \mathrm{rpm}$. The region of existence of stable milling has much more points (shaded area). The dashed line denotes the boundary between stable and unstable milling. In our case the stability boundary corresponds to depth of cut equal to $0.41 \mathrm{~mm}$. The experimental critical value of stable cutting obtained by the RP method is greater than the value obtained by modal analysis and by the software CutPro $(0.3 \mathrm{~mm})$. In fig. 6a, zoom of the recurrence plot for stable milling in the time range from 16 to $18 \mathrm{~s}$ and the unstable one in the time range from 28 to $30 \mathrm{~s}$ (10000 data points) is presented. The recurrence diagram for unstable milling (range time from 28 to $30 \mathrm{~s}$ ) is much more complex and consists of separate points, different length lines and empty spaces (fig. 6b).

The recurrence plot method can be extended to the recurrence quantifications analysis (RQA) based on points and lines in the RP.

\subsection{Recurrence quantifications analysis (RQA)}

The recurrence quantification analysis (RQA) is a method of nonlinear data analysis which quantifies the number and duration of recurrences of a dynamical system presented by its state space trajectory. Zbilut and Webber [16] defined several measures of complexity to quantify the small scale structures of RP. These measures are based on the recurrence points density and the diagonal and the vertical line structures of the recurrence plot. The most important RQA measures are Marwan et al. [15]:

- Recurrence rate $(\mathrm{RR})$, which describes the density of recurrence points. The physical meaning of RR is the probability that the system will recur

$$
R R=\frac{1}{N^{2}} \sum_{i, j=1}^{N} R P_{i, j}
$$



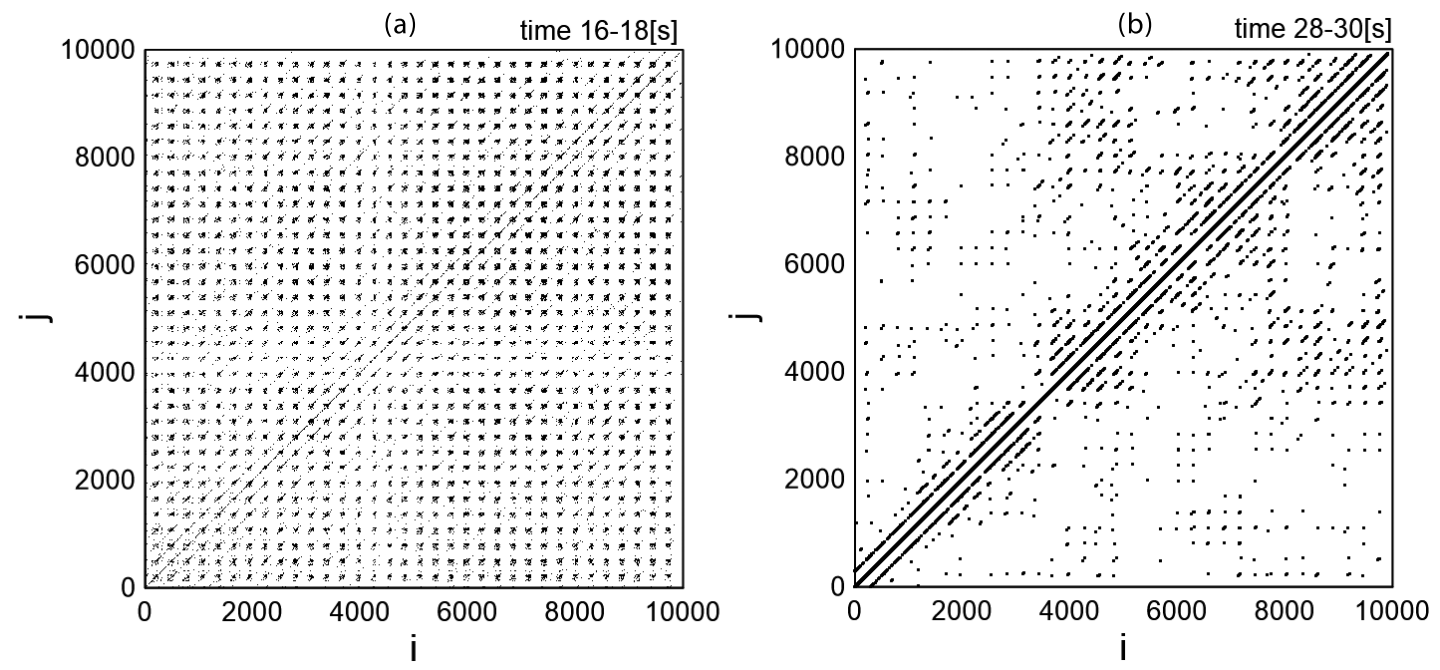

Fig. 6. Recurrence plot for stable milling, $d=9, \varepsilon=10$ (a) and unstable milling, $d=10, \varepsilon=25$ (b) for $m=5$ and 10000 data points.

- Determinism (DET), describes the recurrence points forming diagonal lines. Diagonal lines represent epochs of similar time evolution of states of the system

$$
D E T=\frac{\sum_{l=l_{\min }}^{N} l P(l)}{\sum_{i, j=1}^{N} R P_{i, j}},
$$

- Laminarity (LAM), characterizes of recurrence points forming vertical lines. Vertical lines are typical for intermittency

$$
L A M=\frac{\sum_{\nu=\nu_{\min }}^{N} \nu P(\nu)}{\sum_{\nu=1}^{N} \nu P(\nu)},
$$

- Trapping time (TT), measures the mean time during which the system is trapped in one state or changes only very slowly

$$
T T=\frac{\sum_{\nu=\nu_{\min }}^{N} \nu P(\nu)}{\sum_{\nu=\nu_{\min }}^{N} P(\nu)}
$$

- The length of the longest diagonal and vertical lines can be estimated

$$
L_{\max }=\max \left(\left\{l_{i} ; i=1, \ldots, N_{l}\right\}\right), \quad V_{\max }=\max \left(\left\{l_{\nu} ; i=1, \ldots, N_{\nu}\right\}\right) .
$$

In the above frame RQA provides us with the probability $P(l)$ or $P(v)$ of line distribution according to their lengths in diagonal $(l)$ or vertical $(\nu)$ lines, $N$ is number of points on phase space trajectory. The inverse of $L_{\text {max }}$ (Divergence DIV) is related to the positive Lyapunov exponents Marwan et al. [15]. More detailed description of RP and RQA techniques can be found in [10,17].

All quantifications have been tested in this study and next six of them were selected to better identify milling stability. The first is the RR which measures the ratio between recurrence points to all possible points on the RP diagram. In the unstable region the value of the $R R$ tends to zero (fig. 7a), while the DET/RR ratio is three times higher (fig. $7 \mathrm{~b}$ ). The significantly lower values of $L_{\max }$ (fig. 7c), LAM (fig. 7e), and $V_{\max }$ (fig. $7 \mathrm{f}$ ) show change in dynamics and can be used as stability criterion. However, DIV (fig. 7d) shows a similar behavior as the DET/RR ratio. According to RQA the unstable region begins after $26 \mathrm{~s}$ of the milling test, that corresponds to $a_{p}=0.42 \mathrm{~mm}$. 

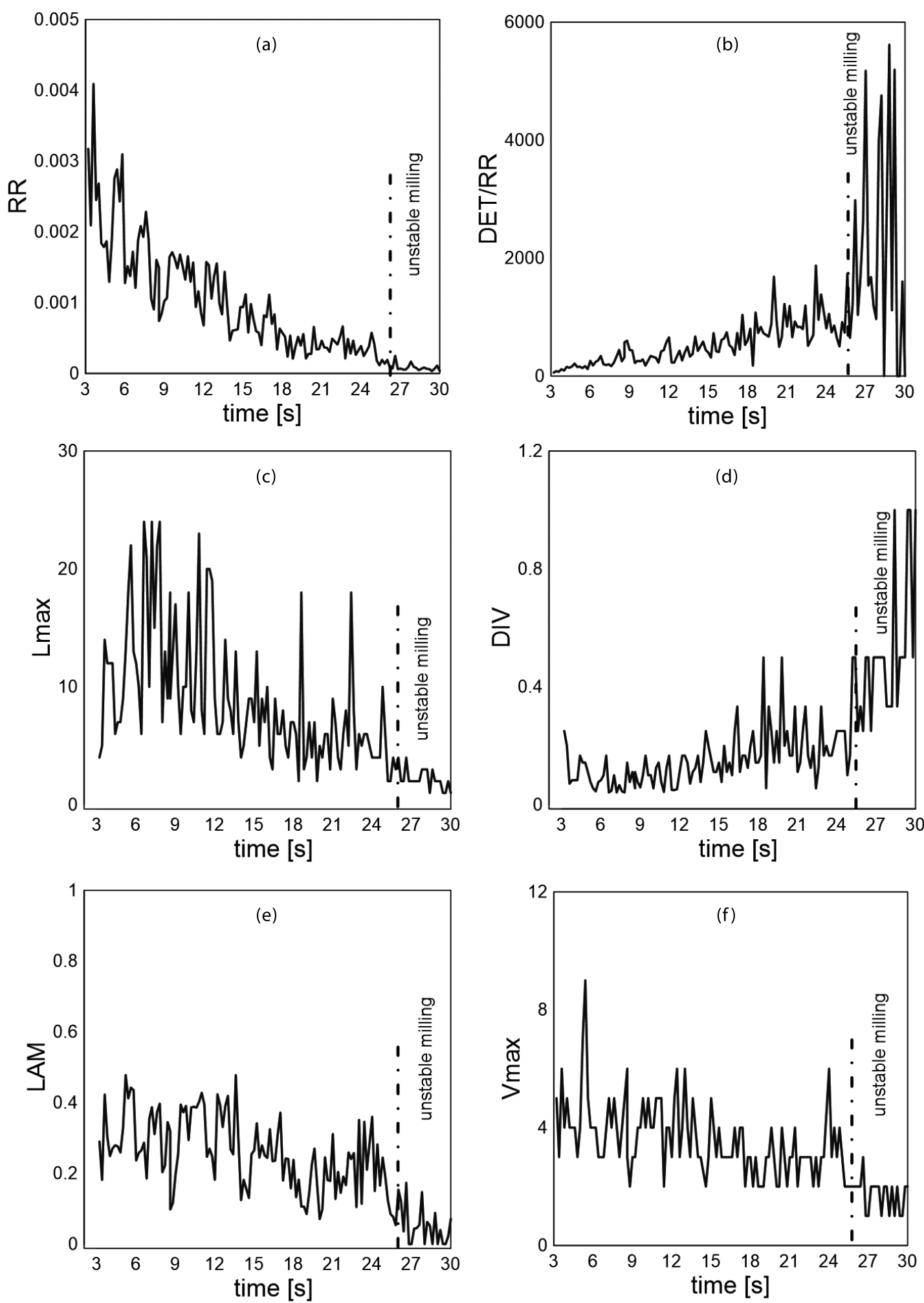

Fig. 7. Selected recurrence quantifications (RQA) used to identify unstable milling process: RR (a), DET/RR ratio (b), $L_{\text {max }}$ (c), DIV (d), LAM (e) and $V_{\max }(\mathrm{f})$. 


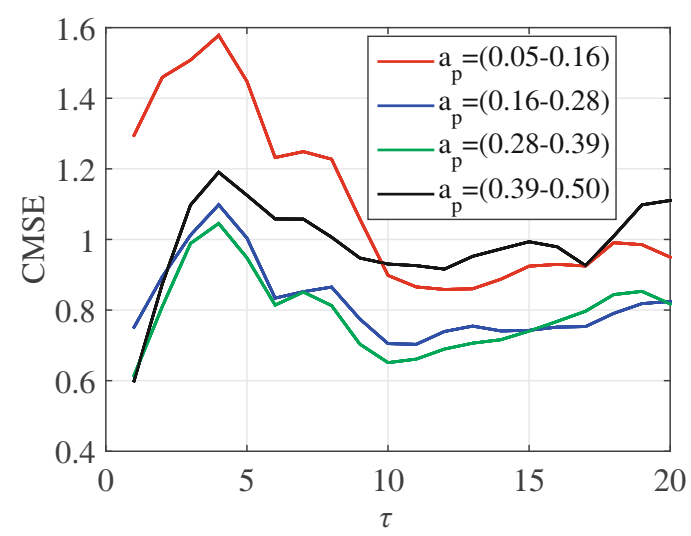

Fig. 8. The composite multiscale entropy analysis calculated for measured forces $F_{x}$ signals for different ranges of the depth of cut $a_{p}$.

\subsection{Multiscale entropy analysis}

A composite multiscale entropy (CMSE) method is another approach to estimate the behavior of the system [18]. In order to perform this analysis, the original time series undergo a coarse-grained procedure, according the formula

$$
y_{k, j}^{(\tau)}=\frac{1}{\tau} \sum_{i=(j-1) \tau+k}^{i=j \tau+k-1} x_{i}, \quad 1 \leq j \leq \frac{N}{\tau}, 1 \leq k \leq \tau .
$$

The coarse-grained time series $\{\mathbf{y}\}$ is averaged on the origin data points within non-overlapping windows by increasing the scale factor $\tau$. For each $\tau$, the composite multiscale entropy calculation is based on the averaged time series $\{\mathbf{y}\}$ and reads as

$$
C M S E(x, \tau, \mu, r)=\frac{1}{\tau} \sum_{k=1}^{\tau} \operatorname{SampEn}\left(y_{k}^{(\tau)}, \mu, r\right),
$$

where $\mu=2$ is the pattern length and $r$ is the similarity criterion and $10 \%$ of the standard deviation of the original time series $\{\mathbf{x}\}$ is adopted. In the above formula the SampEn is the logarithm of the conditional probability that two sequences with a tolerance $r$ are similar to each other at the next points of the coarse-grained series.

The calculated CMSE for cutting force $F_{x}$ at spindle speed $n=1000 \mathrm{rpm}$ is presented in fig. 8 . The measured signal was divided on four windows for the sake of the cut depth $a_{p}$ and the analysis was developed in four separated CMSE results. Figure 8 shows the signal behavior of the force acting along the feed direction $x$. It appears that the most disordered signal behavior seems to be at the lowest depth of cut $a_{p} \approx(0.05-0.16) \mathrm{mm}$ (red line) up to $\tau=10$. For larger scale factor $\tau$, the tendency switches and the signal behaves the most unforeseen at the highest depth of cut $a_{p} \approx(0.39-0.5) \mathrm{mm}$. The other time series windows corresponding to $a_{p} \approx(0.16-0.39) \mathrm{mm}$ seem to be more stable. Interesting phenomena can be observed in fig. 8, the entropy increases initially at scale factors $\tau$ up to $\tau \approx 4$ and next falls down. It can result from the irregularities found for the initially coarse-grained time series which transiently increases the entropy value.

\section{Final conclusions and remarks}

This study focused on the verification of the milling process stability in the case of nickel-chromium superalloy Inconel 718. The stability lobe diagram is determined by the commercial software CutPro (with modal analysis) and the "machinist online" package. Analysis of correctness of the SLD, done by the RP and the RQA methods showed, that the save and real depth of cut $a_{p}$ for $n=1000 \mathrm{rpm}$ is $0.41 \mathrm{~mm}$ (by CutPro9 $0.3 \mathrm{~mm}$, by package "machinist online" $0.1 \mathrm{~mm}$ ).

Among all recurrence quantifications, six have been chosen to identify the unstable process and detect the entry in unstable cutting. The possibility of short time series analysis is a very important advantage of RP and RQA methods. These methods can easily be adopted in the future for monitoring the cutting processes. The independent verification of both the RP and the RQA analysis has been developed by composite multiscale entropy analysis, which confirmed the range of cutting depth at which signal reveals an ordered behavior. In general it is clearly noticed that measured signals of milling forces are the most disordered during the process at relatively small depth of cuts $a_{p}$.

In the near future, a new experiment is being planned for different materials including iron alloys and composites reinforced by glass or carbon fibers. In the future work the authors are going to apply RP and RQA as well as CMSE for results of numerical simulation and to control or monitor the milling process stability. Finally after positive numerical tests, the implementation to a real milling machine is planned. 
Financial support of Structural Funds in the Operational Programme - Innovative Economy (IE OP) financed from the European Regional Development Fund - Project No POIG.0101.02-00-015/08 is gratefully acknowledged.

Open Access This is an open access article distributed under the terms of the Creative Commons Attribution License (http://creativecommons.org/licenses/by/4.0), which permits unrestricted use, distribution, and reproduction in any medium, provided the original work is properly cited.

\section{References}

1. M.J. Jackson, G.M. Robinson, M.D. Whitfield, J. Achiev. Mater. Manufact. Eng. 20, 27 (2007).

2. Y. Jianping, Create a Stability Lobe Diagram, in Proceedings of the International Conference on Engineering 85 Technology, Kean University, New Jersey, 2006.

3. S. Seguy, F.J. Campa, L.N. Lopez de Lacella, L. Arnaud, G. Dessein, G. Armandi, Int. J. Machin. Machin. Mater. 4, 377 (2008).

4. J. Gradisek, E. Govekar, I. Grabec, J. Sound Vibr. 242, 829 (2001).

5. T. Insperger, G. Stepan, P.V. Bayly, B.P. Mann, J. Sound Vibr. 262, 333 (2004).

6. Y.C. Shin, J. Eng. Industry 114, 23 (1992).

7. K. Kecik, R. Rusinek, J. Warminski, J. Phys. Conf. Ser. 382, 012012 (2012).

8. L.N. Lopez de Lacalle, A. Lamikiz, Machine Tools for High Performance Machining (Springer-Verlag, London, 2009).

9. B.P. Mann, T. Insperger, P.V. Bayly, G. Stepan, Int. J. Machine Tools Manufact. 43, 35 (2002).

10. K. Kecik, R. Rusinek, J. Warminski, Int. J. Bifurc. Chaos 21, 1 (2011).

11. J.P. Eckman, S.O. Kamphorst, D. Ruelle, Europhys. Lett. 5, 973 (1987).

12. R. Rusinek, M. Borowiec, Eur. Phys. J. Plus 130, 194 (2015).

13. T. Schmitz, http://highspeedmachining.mae.ufl.edu/.

14. N. Marwan Encounters with Neighbours: Current Development of Concepts Based on Recurrence Plots and Their Applications, PhD Thesis, University of Potsdam (2003).

15. N. Marwan, M.C. Romano, M. Thiel, J. Kurths, Phys. Rep. 438, 237 (2007).

16. J.P. Zbilut, C.L. Jr Webber, Phys. Lett. A 171, 199 (1992).

17. R. Rusinek, Int. J. Non-Linear Mech. 45, 458 (2010).

18. W. Shuen-De, W. Chiu-Wen, L. Shiou-Gwo, W. Chun-Chieh, L. Kung-Yen, Entropy 13, 1069 (2013). 